\title{
Oblique Hanle measurements of InAs/GaAs quantum dot spin-light emitting diodes
}

\author{
G. Itskos, ${ }^{\text {a) }}$ E. Harbord, S. K. Clowes, E. Clarke, L. F. Cohen, and R. Murray \\ Experimental Solid State Physics Group, Blackett Laboratory, Imperial College London, Prince Consort \\ Road, London SW7 2AZ, United Kingdom \\ P. Van Dorpe and W. Van Roy \\ IMEC, Kapeldreef 75, Leuven B-3001, Belgium
}

(Received 28 September 2005; accepted 12 December 2005; published online 11 January 2006)

\begin{abstract}
We report on studies of electrical spin injection from ferromagnetic Fe contacts into semiconductor light emitting diodes containing single layers of InAs/GaAs self-assembled quantum dots (QDs). An oblique magnetic field is used to manipulate the spin of the injected electrons in the semiconductor. This approach allows us to measure the injected steady-state spin polarization in the QDs, $P_{\text {spin }}$ as well as estimate the spin losses in the QD spin detector. After subtraction of magneto-optical effects not related to spin injection, we measured a $P_{\text {spin }}$ of $7.5 \%$ at $15 \mathrm{~K}$ and estimated an injected spin polarization before QD recombination of around 20\%. (C) 2006 American Institute of Physics. [DOI: 10.1063/1.2163074]
\end{abstract}

Electrical injection of spin polarized carriers from a magnetic contact into a semiconductor is essential for the implementation of spintronic devices. ${ }^{1,2}$ An accurate way to quantify the spin injection process is to utilize a spin-light emitting diode (spin-LED). Such a device uses a magnetic contact to electrically inject spin polarized carriers into a LED structure and employs the radiative selection rules to relate the degree of the circular polarization of the emitted light to the injected carrier spin polarization. Ferromagnetic metals are attractive as magnetic contacts in spin-LEDs due to their high Curie temperatures and their well-studied structural and magnetic properties. Recently, high spin injection efficiencies have been achieved in devices where a conventional ferromagnetic metal such as $\mathrm{Fe}, \mathrm{Co}, \mathrm{Ni}$ and their alloys were used to electrically inject spin-polarized electrons via a Schottky ${ }^{3-5}$ or an oxide tunnel barrier ${ }^{6-9}$ into a semiconductor LED structure.

In the great majority of these devices, bulk semiconductors or quantum wells have been used as the recombination region. Spin relaxation in these is mainly caused by momentum-dependent spin interactions that are in general fast because of the availability of a continuum of energy states. These mechanisms should be inefficient in spin-LEDs based on quantum dots (QDs) due to their three-dimensional carrier confinement, which results in a discrete density of states. Yet, there have been few reports on electrical spin injection in QD-based spin-LEDs, ${ }^{10-12}$ only one of which ${ }^{12}$ used a ferromagnetic contact as injector. In the latter, a large magnetic field $(B>2 \mathrm{~T})$ was used to bring the Fe film magnetization out of plane (hard axis) and inject electrons with spins along the surface normal (Faraday geometry). An injected spin polarization in the QDs, $P_{\text {spin }}$ of $5 \%$ was measured; importantly the polarization was insensitive to the temperature and persisted to $300 \mathrm{~K}$, confirming the potential of quantum dots as spin detectors in spin-LEDs.

We demonstrate here electrical spin injection from ferromagnetic $\mathrm{Fe}$ contacts into semiconductor LED structures containing single layers of InAs/GaAs self-assembled quan-

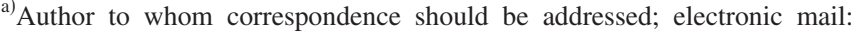
g.itskos@imperial.ac.uk tum dots (QDs). The spins are injected along the sample layers (Fe easy axis) under application of a small oblique magnetic field $(\mathrm{B}<100 \mathrm{mT})$. The oblique Hanle effect ${ }^{13}$ allow us to measure the injected spin polarization in the QDs, estimate the spin scattering and gain information on the QD spin dynamics.

The LED structures were grown by molecular beam epitaxy on $p$-GaAs (001) substrates. A $1000 \mathrm{~nm} p$-doped GaAs buffer $\left(p=10^{18} \mathrm{~cm}^{-3}\right)$ was deposited at $580{ }^{\circ} \mathrm{C}$ followed by $200 \mathrm{~nm}$ of a $p$-doped $\mathrm{Al}_{0.3} \mathrm{Ga}_{0.7}$ As blocking layer. The intrinsic (i) region of the $p-i-n$ structure consisted of $25 \mathrm{~nm}$ of undoped GaAs grown at $580^{\circ} \mathrm{C}$ which was annealed for 10 min before the substrate temperature was reduced to $495{ }^{\circ} \mathrm{C}$ for the growth of the QD layer. The QDs were formed by the deposition of $2.4 \mathrm{ML}$ of InAs, capped by $25 \mathrm{~nm}$ of undoped $\mathrm{GaAs}$ at $495{ }^{\circ} \mathrm{C}$. The growth temperature was then raised to $580{ }^{\circ} \mathrm{C}$ for the deposition of a $60 \mathrm{~nm} \mathrm{Al} l_{0.1} \mathrm{Ga}_{0.9}$ As contact layer. The doping profile of this layer was chosen so that it forms a thin triangular shaped Schottky barrier to the Fe overlayer. The structure was capped with a layer of $\mathrm{Sb}$ to prevent oxidation during transfer to a second molecular beam epitaxy (MBE) machine where after the removal of the $\mathrm{Sb}$ cap a $10-\mathrm{nm}$-thick $\mathrm{Fe}$ contact was epitaxially grown at $0{ }^{\circ} \mathrm{C}$. The Fe layer was then covered by a $5 \mathrm{~nm}$ GaAs cap to protect it from oxidation. The samples were processed into surface emitting LEDs using conventional optical lithography, dry and wet processing steps.

The spin-LED cross section and the flatband diagram of the device are depicted in Fig. 1. The structure consists of a combination of two diodes, a Schottky formed by the $\mathrm{Fe} / \mathrm{AlGaAs}(n)$ barrier and an $\mathrm{Al}(\mathrm{Ga}) \mathrm{As} / \mathrm{QDs} n-i-p$ diode, connected back to back. Under the bias conditions of Fig. 1, the Schottky diode is reverse biased to allow efficient tunneling of electrons from the $\mathrm{Fe}$ into the semiconductor while the $n-i-p$ diode is forward biased to ensure collection of injected electrons and unpolarized holes from the $p$ region, into the QDs. Light emission is attributed to excitonic recombination and occurs for bias voltages larger than $1.65 \mathrm{~V}$.

In our samples, the magnetization of the thin Fe contact lies in plane due to shape anisotropy. Under bias, spins are injected into the semiconductor along the easy axis of the 


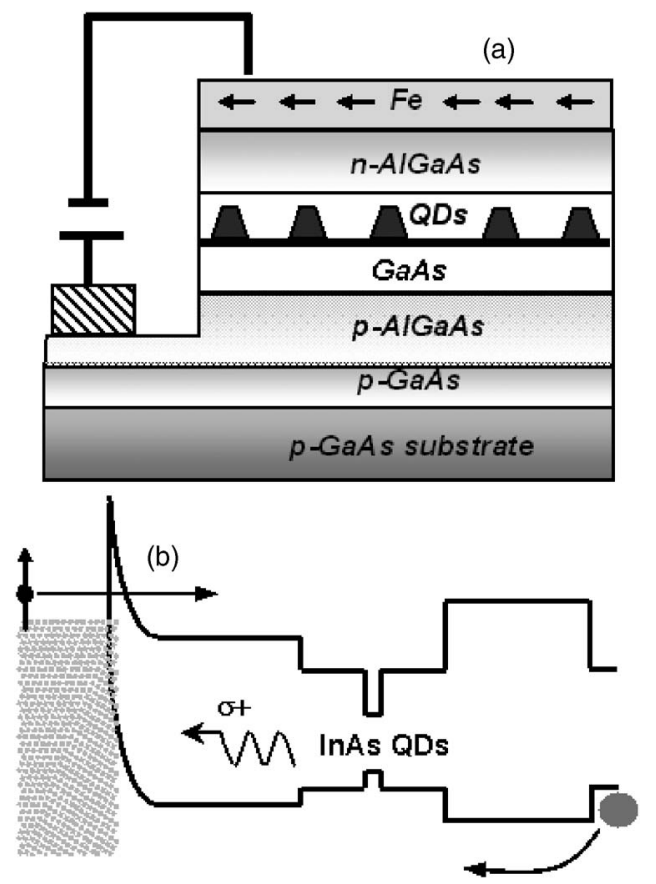

FIG. 1. (a) Spin-LED schematic cross section. (b) Flatband diagram of the device.

ferromagnet $\left(\mathbf{S}_{\mathbf{o y}}=\mathbf{S}_{\mathbf{o y}} \cdot \mathbf{y}\right.$ where $\mathbf{y}$ denotes the easy axis direction). When an oblique magnetic field $\mathbf{B}$ is applied, the injected spins start to precess around it with a Larmor frequency $\boldsymbol{\Omega}=g^{*} \mu_{B} \mathbf{B} / \hbar$ (see inset of Fig. 2) where $g^{*}$ is the effective Landé factor. The average spin $\mathbf{S}$ is then aligned along $\mathbf{B}$ giving a nonzero component along the sample normal $\mathbf{S}_{\mathbf{z}}$, which according to the radiative selection rules ${ }^{14}$ results in the emission of circular polarized light. It was recently shown ${ }^{15}$ that there is efficient conversion of spin to photon polarization in QDs along the growth direction which is the geometry employed here.

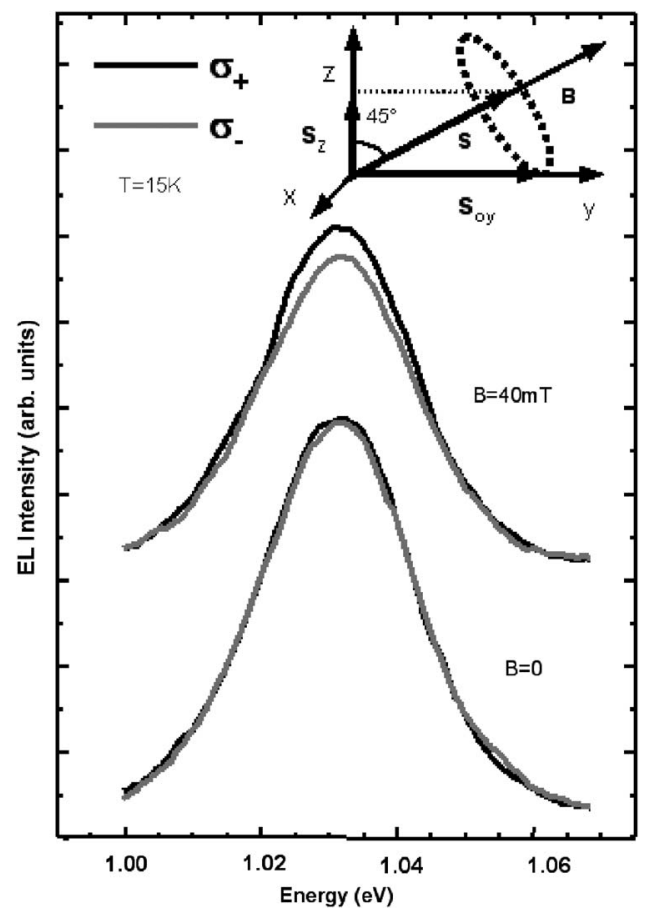

FIG. 2. Electroluminescence spectra in the vicinity of the QD ground exciton emission at $T=15 \mathrm{~K}$ under a bias of $2.5 \mathrm{~A} / \mathrm{cm}^{2}, V=2.1 \mathrm{~V}$ and analyzed for $\sigma_{+}$and $\sigma_{-}$polarizations. A schematic diagram of the oblique Hanle effect

shows the opposite sign of the polarization exhibited by the
is also included (top).
Downloaded 03 Jun 2009 to 131.227.178.130. Redistribution subject to AlP license or copyright; see http://apl.aip.org/apl/copyright.jsp

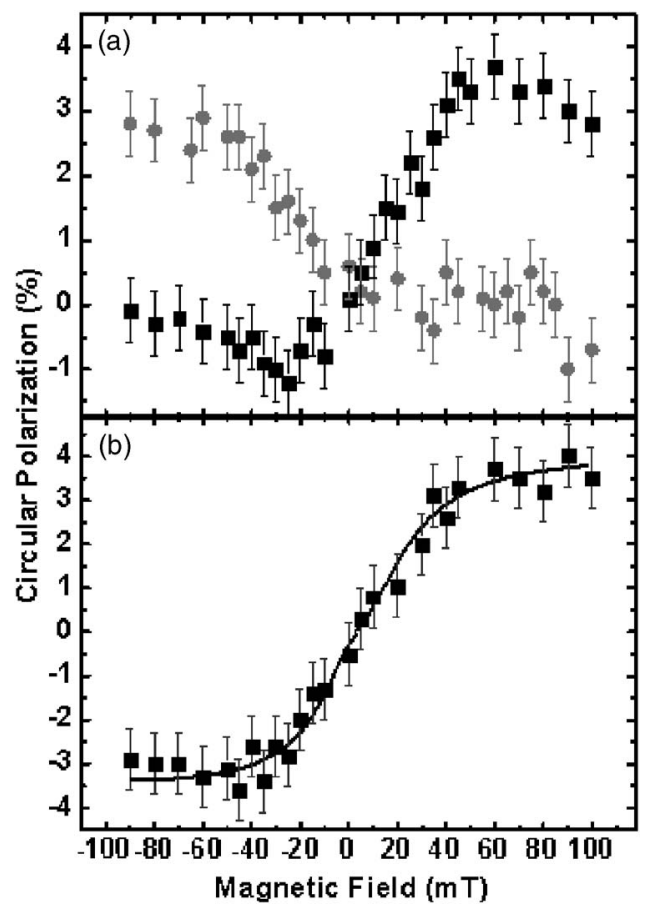

FIG. 3. (a) Circular polarization of the QD ground state EL emission (black square points) and the corresponding PL emission excited with unpolarized light (gray circle points) vs applied oblique magnetic field. (b) Circular polarization after subtraction of nonspin injection related effects. The error bars represent the error on the polarization measurement due to the limitations of the achromatic waveplate/linear polarizer used.

Figure 2 shows electroluminescence spectra in the vicinity of the QD ground exciton emission at $T=15 \mathrm{~K}$, analyzed for $\sigma_{+}$and $\sigma_{-}$polarizations. The narrow linewidth (full width at half maximum $=22 \mathrm{meV}$ ) and long emission wavelength $(1.031 \mathrm{eV})$ is a consequence of the low QD growth rate used. ${ }^{16}$ At zero applied magnetic field the $\sigma_{+}$and $\sigma_{-}$components of the QD ground state exciton are almost equal, since there is no component of the electrons spin normal to the surface. When a small magnetic field is applied at $45^{\circ}$ to the sample normal, a nonzero spin component $S_{z}$ is induced that results in a measurable circular polarization of the electroluminescence. The sign of the circular polarisation $\left(\sigma_{+}\right)$is consistent with injection of majority spins from the $\mathrm{Fe}$.

The circular polarisation $P_{\text {circ }}$ of the QD ground state exciton versus applied oblique $\left(45^{\circ}\right)$ magnetic field $B$ is displayed in Fig. 3(a) (black square symbols). The curve is nonlinear, with $P_{\text {circ }}$ rapidly increasing up to $40-50 \mathrm{mT}$ and then slightly decreasing for higher $B$ values. The slight asymmetry of the data behavior between positive and negative magnetic fields is attributed to a small underestimation of the field values due to limitations of the magnet calibration at very low fields. In the same figure, the results of a photoluminescence (PL) study exciting with an unpolarized $\mathrm{He}-\mathrm{Ne}$ $(1.96 \mathrm{eV})$ laser source, are shown (gray circular symbols). Excitation with unpolarized light creates a population of unpolarized electrons and holes in the quantum dots. The circular polarization of the emitted light then quantifies the contribution of nonspin injection effects such as the magnetooptical circular dichroism induced by the Fe film and the QD exciton Zeeman splitting. The PL polarization exhibits a nearly linear behavior with field with no evident tendency towards saturation at higher magnetic fields; moreover, it shows the opposite sign of the polarization exhibited by the 
spin-LED emission. The effect of the tilting of the Fe film magnetization due to the oblique magnetic field is also considered. Based on the analysis of Ref. 7, a negligible tilting angle of less than $2^{\circ}$ is obtained due the small magnitude $(B<100 \mathrm{mT})$ of the external magnetic field.

By subtracting the results of the PL control experiment from those obtained in the spin-LEDs, the circular polarization due to spin-injection effects only is obtained [Fig. 3(b)]. The curve is nonlinear and can be fitted within the framework of the oblique Hanle effect by the Lorentzian function ${ }^{7}$

$\mathbf{S}_{\mathbf{z}}(\mathbf{B})=\mathbf{S}_{\mathbf{o y}} \cdot\left(T_{S} / \tau\right) \cdot\left[\left(\boldsymbol{\Omega} T_{S}\right)^{2} \cos \varphi \sin \varphi\right] /\left[1+\left(\boldsymbol{\Omega} T_{S}\right)^{2}\right]$.

Here $T_{S}$ is the QD ground exciton spin lifetime defined by the exciton spin relaxation time $\tau_{S}$ and the exciton recombination lifetime $\tau\left(T_{S}^{-1}=\tau_{S}^{-1}+\tau^{-1}\right)$. According to the selection rules, ${ }^{14} \mathbf{S}_{\mathbf{z}}(B)$ is simply equal to the circular polarization $P_{\text {circ }}(B)$ of the excitonic recombination in the quantum dots, assuming that the degeneracy of the heavy and light hole bands is lifted by the confinement/strain.

Good fits to the data are obtained using [Fig. 3(b)] two single Lorentzians, one for positive (half width $B_{1 / 2}$ $=24 \pm 2.5 \mathrm{mT})$ and one for negative $\left(B_{1 / 2}=19 \pm 1.5 \mathrm{mT}\right)$ fields due to the anisotropy mentioned earlier. Using an average value $B_{1 / 2}$ of $21.5 \pm 2.9 \mathrm{mT}$, we derive a scaled ground state exciton spin lifetime $g^{*} T_{s}=\hbar / \mu_{B} B_{1 / 2}=510 \pm 70 \mathrm{ps}$, which is approximately two times higher than $g^{*} T_{s}$ values obtained in optical Hanle measurements using quasiresonant laser excitation. ${ }^{17,18}$ The enhancement could be related to the long wavelength emission of our QDs that ensures a large energy difference between the lowest QD states and the InAs wetting layer and is likely to reduce exciton spin dephasing.

From the saturation value $S_{z \max }$ of the Hanle curve, we obtain an injected spin polarization from the Fe film to the semiconductor, before recombination in the QDs of $\Pi=S_{o y}$ $=(7.5 \pm 0.7) \% \cdot \tau / T_{S}$. The $7.5 \%$ value is the measured steadystate spin polarization in the QDs, $P_{\text {spin }}$, due to spin injection effects. The ratio $T_{S} / \tau$ describes the exciton spin scattering in the dots and is estimated to be equal to $0.375 \pm 0.05$, using the previously obtained value of $510 \mathrm{ps}$ for $g^{*} T_{S}$, a ground state exciton lifetime $\tau$ of $800 \mathrm{ps}$, from time-resolved studies in our InAs $\operatorname{dots}^{19}$ and a $g^{*}$-factor estimate of -1.7 . A $\Pi$ value of $20 \pm 3.25 \%$ is then obtained; we note that a correct estimate of $\Pi$ depends on a correct estimate of the $g^{*}$ factor. The $g^{*}$ factor used was obtained from measurements on dots with similar size and emission energy as ours $;{ }^{20}$ the negative sign is consistent with the variation of the optical polarization with magnetic field observed in the PL control experiment.

$\Pi$ values of the same order were reported in similar GaAs bulk-based spin-LED structures ${ }^{7}$ this indicates that incorporation of the dot layer in the spin-LED structure does not substantially affect the spin injection process from the metal to the semiconductor. On the other hand, the relatively small value of the measured QD spin polarization $P_{\text {spin }}$ indicates a high exciton spin scattering after capturing, relaxation and recombination of the carriers in the QD layer (scattering ratios $T_{S} / \tau$ of half the magnitude have been measured in bulk GaAs-based spin-LEDs $\left.{ }^{7}\right) . P_{\text {spin }}$ values of the the same order (5\%) have been obtained from Faraday geometry measurements ${ }^{12}$ in similar spin-LED structures. We note here that the two efficiencies are not directly comparable but rather are complementary; the spin relaxation rate depends on the magnitude and direction of the applied magnetic field ${ }^{14,21}$ which are markedly different in the OHE and Faraday geometries.

In summary, electrical spin injection from Fe films into InAs QD-based spin-LEDs has been investigated using the oblique Hanle effect. We have measured an injected spin polarization $P_{\text {spin }}$ in the QDs of 7.5\% at low temperatures and obtain information on QD exciton spin dynamics (half width $B_{1 / 2}$ and scaled ground state exciton spin lifetime). Based on those values, the spin injected polarization from the Fe film into the semiconductor $\Pi$ was estimated to be around $20 \%$ indicating a substantial spin loss in our spin detector. Optimization of the dot layer, using, for example, positively charged $\mathrm{QDs}^{22}$ that exhibit larger spin relaxation times and shorter recombination times could result in improved $P_{\text {spin }}$ values. The demonstrated electrical injection of spins at small magnetic fields $(<100 \mathrm{mT})$ along with the potential for room temperature operation ${ }^{12}$ makes QD-based spin-LEDs promising candidates for the realization of practical spintronic devices.

The authors thank W. van de Graaf and S. Degroote for MBE sample growth; P.V.D. acknowledges financial support from the IWT (Flanders). This work is partly supported by the EC project FENICS (G5RD-CT-2001-00535). E.H. would like to thank EPRSC for a studentship.

${ }^{1}$ M. Oestreich, Nature (London) 402, 735 (1999).

${ }^{2}$ I. Zutic, J. Fabian, and S. Das Sarma, Phys. Rev. B 64, 121201 (2001).

${ }^{3}$ A. T. Hanbicki, B. T. Jonker, G. Itskos, G. Kioseoglou, and A. Petrou, Appl. Phys. Lett. 80, 1240 (2002).

${ }^{4}$ C. Adelmann, J. Q. Xie, C. J. Palmstrøm, J. Strand, X. Lou, J. Wang, and P. A. Crowell, J. Vac. Sci. Technol. B 23, 1747 (2005).

${ }^{5}$ A. T. Hanbicki, O. M. J. van't Erve, R. Magno, G. Kioseoglou, C. H. Li, B. T. Jonker, G. Itskos, R. Mallory, M. Yasar, and A. Petrou, Appl. Phys. Lett. 82, 4092 (2003).

${ }^{6}$ V. F. Motsnyi, J. De Boeck, J. Das, W. Van Roy, G. Borghs, E. Goovaerts, and V. I. Safarov, Appl. Phys. Lett. 81, 265 (2002).

${ }^{7}$ V. F. Motsnyi, P. Van Dorpe, W. Van Roy, E. Goovaerts, V. I. Safarov, G. Borghs, and J. De Boeck, Phys. Rev. B 68, 245319 (2003).

${ }^{8}$ O. M. J. van't Erve, G. Kioseoglou, A. T. Hanbicki, C. H. Li, B. T. Jonker, R. Mallory, M. Yasar, and A. Petrou, Appl. Phys. Lett. 84, 4334 (2004).

${ }^{9}$ X. Jiang, R. Wang, R. M. Shelby, R. M. Macfarlane, S. R. Bank, J. S. Harris, and S. S. P. Parkin, Phys. Rev. Lett. 94, 056601 (2005).

${ }^{10}$ Y. Chye, M. E. White, E. Johnson-Halperin, B. D. Gerardot, D. D. Awschalom, and P. M. Petroff, Phys. Rev. B 66, 201301(R) (2002).

${ }^{11}$ S. Ghosh and P. Bhattacharya, Appl. Phys. Lett. 80, 658 (2002). Note that there can be no direct measurement of the spin injection efficiency in this letter; the selection rules that relate the spin injection efficiency to the emission circular polarization do not apply for the geometry employed.

${ }^{12}$ C. H. Li, G. Kioseoglou, O. M. J. van't Erve, M. E. Ware, D. Gammon, R. M. Stroud, B. T. Jonker, R. Mallory, M. Yasar, and A. Petrou, Appl. Phys. Lett. 86, 132503 (2005).

${ }^{13}$ M. I. D’yakonov, V. I. Perel, V. L. Berkovits, and V. I. Safarov, Sov. Phys. JETP 40, 950 (1975).

${ }^{14}$ F. Meier and B. P. Zachachrenya, Optical Orientation (North-Holland, Amsterdam, 1984).

${ }^{15}$ C. E. Pryor and M. E. Flatté, Phys. Rev. Lett. 91, 257901 (2003).

${ }^{16}$ P. B. Joyce, T. J. Krzyzewski, G. R. Bell, T. S. Jones, E. C. Le Ru, and R. Murray, Phys. Rev. B 64, 235317 (2001).

${ }^{17}$ R. J. Epstein, D. T. Fuchs, W. V. Schoenfeld, P. M. Petroff, and D. D. Awschalom, Appl. Phys. Lett. 78, 733 (2001).

${ }^{18}$ J. Fu/rsst, H. Pascher, V. A. Abalmassov, T. S. Shamirzaev, and K. S. Zhuravlev, Semicond. Sci. Technol. 20, 209 (2005).

${ }^{19}$ S. Malik, E. C. Le Ru, D. Childs, and R. Murray, Phys. Rev. B 63, 155313 (2001).

${ }^{20}$ T. Nakaoka, T. Saito, J. Tatebayashi, and Y. Arakawa, Phys. Rev. B 70, 235337 (2004).

${ }^{21}$ D. V. Bulaev and D. Loss, Phys. Rev. B 71, 205324 (2005).

${ }^{22}$ K. Gündoğdu, K. C. Hall, T. F. Boggess, D. G. Deppe, and O. B. Shchekin, Appl. Phys. Lett. 84, 2793 (2005). 